\title{
Displacement Sensor Based on Diamond-Shaped Tapered Split Ring Resonator
}

\author{
Ali Karami Horestani, Student Member, IEEE, Christophe Fumeaux, Senior Member, IEEE, \\ Said F. Al-Sarawi, Member, IEEE, and Derek Abbott, Fellow, IEEE
}

\begin{abstract}
Split-ring resonators (SRRs) are ideal structures for the realization of compact high-sensitivity and high-resolution sensors due to their high-quality factor resonance, compact size, and high sensitivity to changes in the constituent materials and physical dimensions. This paper presents a displacement sensor based on a diamond-shaped tapered SRR coupled to a coplanar waveguide. Two significant improvements over previous designs are reported. Firstly, the proposed sensor has higher dynamic range and linearity for displacement sensing. Secondly, compared with previous designs, where the displacement changes both the resonant frequency and depth of the transmission notch, the proposed sensor has a fixed resonant frequency. This is an important improvement since the sensor can be operated at a single fixed frequency and bypass the need for a frequencysweeping microwave source and measurement system such as an expensive network analyzer. It is shown that, while preserving the compact size, the proposed sensor also benefits from a lower operating frequency. The design principle and simulation results are validated through measurement.
\end{abstract}

Index Terms-Displacement sensor, metamaterials, microwave sensors.

\section{INTRODUCTION}

$\mathbf{M}$ ETAMATERIALS are artificially engineered materials composed of electrically small particles that are able to create a specified material's effective permittivity and/or permeability [1]. Recently, the concept of metamaterials has attracted great interest due to the unusual properties of these artificial materials in microwave, millimetre-wave and optical frequency bands [2]. With the significant possibility of tailoring their constitutive parameters, metamaterials have opened new perspectives for many novel applications such as cloaking [3], [4], directive antennas [5], transparent devices [6], high-quality resonators [7], compact filters [8] or perfect lenses [9].

It has been also shown that resonators such as the splitring resonator (SRR), inspired by metamaterials, can be

Manuscript received March 27, 2012; revised August 15, 2012; accepted November 14, 2012. Date of publication November 30, 2012; date of current version February 5, 2013. The work of C. Fumeaux was supported by the ARC Future Fellowship funding scheme under FT100100585.

A. K. Horestani is with the School of Electrical and Electronic Engineering, The University of Adelaide, Adelaide 5005, Australia, and also with the Ministry of Science, Research and Technology, Tehran 16846-13114, Iran (e-mail: akarami@eleceng.adelaide.edu.au).

C. Fumeaux, S. F. Al-Sarawi, and D. Abbott are with the School of Electrical and Electronic Engineering, The University of Adelaide, Adelaide 5005, Australia (e-mail: cfumeaux@eleceng.adelaide.edu.au; alsarawi@eleceng.adelaide.edu.au; dabbott@eleceng.adelaide.edu.au).

Color versions of one or more of the figures in this paper are available online at http://ieeexplore.ieee.org.

Digital Object Identifier 10.1109/JSEN.2012.2231065 used in the design of high sensitivity and high resolution sensors [10]-[12]. In fact, the high quality factor resonance, subwavelength dimensions, and the sensitivity of the resonance to the constituent materials and physical dimensions of the SRR make it an ideal structure for sensing applications [13]. Furthermore, it has been shown that the sensitivity of the conventional sensors can be dramatically enhanced by using metamaterial structures [2].

According to these unique properties, Zheludev [14] pointed out that sensory devices open another promising area for the future application of metamaterials. This is evidenced by the increasing number of publications proposing the application of metamaterial particles in various types of sensors such as chemical sensing with enhanced sensitivity [15], biosensing [16], enhanced molecular detection techniques based on metamaterials [17], [18], metamaterial-based wireless strain sensors [19], dielectric sensing [20] or structural defects detectors based on metamaterial lenses [21].

This article presents a displacement sensor based on a modified SRR coupled to a coplanar waveguide (CPW). The motivation for such a sensor over traditional methods (e.g. optical interferometry) is that it is low-cost, passive and hence not sensitive to environmental variations such as temperature, and easily integrable with, say, MEMS technology. The basic principle of displacement sensing based on SRRs is first briefly outlined in the next section. Two significant improvements over previous designs are then proposed in Section III. Firstly, a diamond-shaped SRR is introduced to increase the sensor's linearity and dynamic range of displacement sensing. Secondly, compared to a previous design [22], where the displacement changed both the resonant frequency and depth of the bandstop notch, a tapered shape SRR is introduced here to keep a fixed resonant frequency. This is an important improvement since the sensor does not require a frequency sweeping microwave source such as an expensive network analyzer, but can be operated at a single frequency. It is also demonstrated that, while retaining a compact size, the proposed sensor benefits from a lower operating frequency. A physical explanation involving the slot line mode is provided in Section IV. The design principle is validated through measurements in Section V, followed by the concluding remarks.

\section{BASIC OF SRR-BASED DisplaCEMENT SENSOR}

Further to applications in extended array in metamaterial media, single SRRs can be used along with planar transmission lines (TLs) in filter applications [23], [24]. An SRR coupled to 


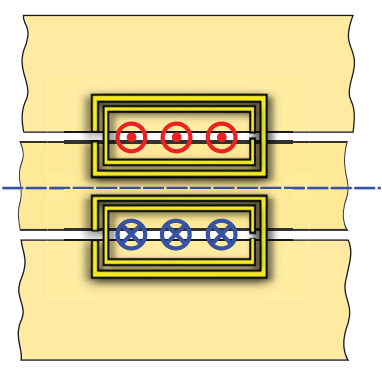

(a)

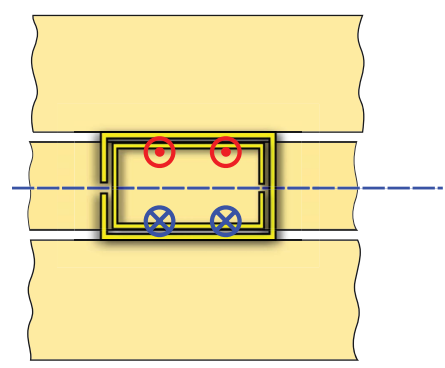

(b)

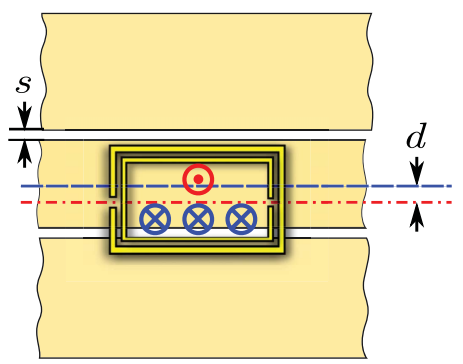

(c)

Fig. 1. Bottom view of a coplanar waveguide loaded with SRRs. (a) Typical configuration in filter applications, which involves a pair of double-ring SRRs under the CPW slots. (b) One double-ring SRR aligned with CPW symmetry plane. (c) One double-ring SRR displaced by $d$ from the symmetry plane of the CPW. (Adapted from [22].)

a TL can inhibit the propagation of the electromagnetic wave along the TL in the vicinity of the SRR resonant frequency. This introduces a notch in the transmission spectrum that can be interpreted as due to the negative effective permeability of the structure [25]. Metamaterial TLs and their sensitivity to slight environmental changes in their vicinity can be exploited in various types of sensors such as thin-film sensors [13], [26].

In order to implement SRR-based sensors two main strategies have been used, both based on alteration of the resonance properties caused by the variable to be sensed: Firstly, sensing can be based on changes of the resonant frequency of the SRR, and secondly, it can use the variation of the quality factor of the resonance [13]. Recently, displacement and rotation sensors based on the variation in the depth of notch in the transmission spectrum have been proposed by Naqui et al. [22]. The operation of these sensors relies on the loss of symmetry, which is caused by a displacement or rotation of an SRR coupled to a coplanar waveguide (CPW).

A typical configuration of SRR loaded CPW consists of a pair of double ring SRRs etched on the back side of the CPW's substrate with the centre of double ring SRRs symmetrically located underneath the two slots of the CPW. The SRRs, which are magnetically coupled to the CPW, inhibit the electromagnetic wave propagation along the CPW in a narrow band in the vicinity of their resonant frequency [25]. As shown in Fig. 1.(a), in this case the magnetic fields passing through the surfaces of the two symmetrical SRRs are contradirectional.

In order to implement a displacement sensor, Naqui et al. [22] used only a double ring rectangular SRR etched on the back side of the CPW substrate, and centred below the CPW line, instead of two SRRs aligned with the CPW's slots. In the initial position, shown in Fig. 1.(b), the SRR is therefore perfectly aligned with the symmetry line of the CPW transmission line. Under these conditions, since the total magnetic flux passing through the surface of the SRR is the vector summation of the fluxes coming from both CPW's slots, the total magnetic flux passing through the SRR surface is zero. As a consequence, a symmetrically aligned SRR cannot be excited at resonance, and consequently there is no spectral notch in the structure's transmission coefficient. However, if the symmetry is broken by a lateral displacement $d$ of the SRR, as shown in Fig. 1.(c), a net magnetic flux through the surface of the SRR appears. Thus, a transmission zero at the resonant frequency of the SRR is expected. Since the depth of the transmission notch depends on the deviation of the SRR from the symmetry plane, the amount of the displacement $d$ can be sensed from the magnitude of such transmission zero [22].

\section{Displacement Sensor Based ON DIAMOND-SHAPED SRR}

In spite of the advantages of the proposed displacement sensor in [22] such as high sensitivity for small values of displacement, the sensor has some limitations, namely, a limited dynamic range and a displacement-dependent shift in the resonant frequency. The first part of this section proposes a diamond-shaped SRR, which increases the dynamic range and improves the linearity of the sensor, while the second subsection addresses the shift in the resonant frequency.

\section{A. Dynamic Range and Linearity ${ }^{1}$}

The limited dynamic range and non-linearity of the displacement sensor based on a rectangular SRR can be explained by the geometry. At first, for small values of the displacement of the rectangular-shaped SRR from the symmetry plane of the CPW, there is an increase in the amount of the net magnetic flux passing through the SRR surface. However, while further increasing the displacement, the net magnetic flux through the SRR surface reaches its maximum when the SRRs horizontal side passes the CPW slot. Beyond this point, as demonstrated by simulation and measurement results in [22], the displacement sensor goes into saturation, and only a minor increase in the notch magnitude can be observed. In fact, as will be shown later in this section, the linear dynamic range of the displacement sensor based on the rectangularshaped SRR is approximately in the order of the CPW slot's width, $s$.

In order to address the non-linearity and dynamic range limitations, a diamond-shaped SRR, shown in Fig. 2, is proposed. In this case, the magnetic flux through the SRR surface becomes maximal when the SRR horizontal diagonal is aligned with the CPW's slot. Thus, in contrast to the rectangular geometry, the angle between the sides of the diamondshaped SRR and the slots of the CPW makes the spatial

\footnotetext{
${ }^{1}$ Note that in this article "linearity" refers to the linearity of $S_{21}$ in "dB" versus the displacement of the SRR.
} 


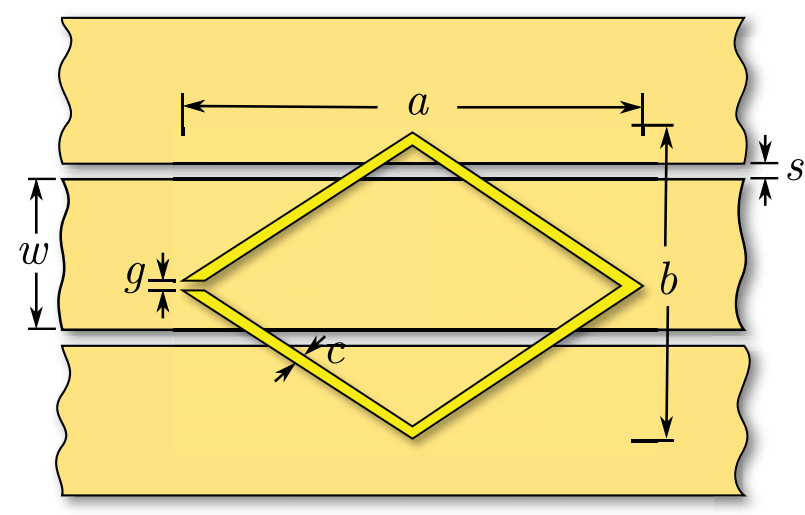

Fig. 2. Bottom view of coplanar waveguide loaded with a diamond-shaped SRR.

dynamic range of the diamond-shaped sensor dependent on the width of the CPW's signal strip (approximately half the width of the signal strip). Therefore, a diamond-shaped SRR coupled to a wide CPW can be used to increase the dynamic range and linearity of the displacement sensor. It is worth recalling that simultaneous increase of the width of the CPW's signal strip and its slots maintains the desired $50 \Omega$ characteristic impedance.

Fig. 2 depicts the bottom view of the proposed displacement sensor, which is composed of a diamond-shaped SRR coupled to a CPW with a relatively wide signal strip. For a specific design performed using 3D electromagnetic simulations, a $0.127 \mathrm{~mm}$ thick Rogers RO3010 material with relative permittivity of 10.2 and copper thickness of $17 \mu \mathrm{m}$ is used as the substrate. The CPW's signal trace width $w$ is $3.1 \mathrm{~mm}$ and the slots' width is $s=0.3 \mathrm{~mm}$, which correspond to a $50 \Omega$ characteristic impedance. The horizontal and vertical diagonals of the diamond-shaped SRR are $a=10 \mathrm{~mm}$ and $b=6 \mathrm{~mm}$, respectively. Finally, the width of the metal strips of the SRR $c$ as well as the SRR's gap $g$ are set to $0.2 \mathrm{~mm}$.

It is worth mentioning that the sensitivity of the sensor not only depends on the geometry of the SRR, but is also affected by the coupling between the SRR and the CPW. Thus, in order to have maximum coupling between SRR and $\mathrm{CPW}$, and consequently maximize the sensor's sensitivity, a thin substrate with $0.127 \mathrm{~mm}$ thickness and high relative permittivity $\left(\epsilon_{r}=10.2\right)$ is selected. However, using a thin substrate results in weak inter-coupling between the inner and outer rings of the SRR, and since there is a small difference between the length of the inner and outer rings, the second resonance is very close to the fundamental resonance of the SRR. In order to avoid the effect of the second resonance a single ring SRR, as shown in Fig. 2, is used instead of a double ring SRR.

Fig. 3 depicts the simulated transmission coefficient of the sensor based on the diamond-shaped SRR for the SRR displacement $d$ varying between $0 \mathrm{~mm}$ and $1 \mathrm{~mm}$ in steps of $0.2 \mathrm{~mm}$. As clearly observed from the figure, an increase in the displacement of the SRR leads to an increase in the notch magnitude, which can be used to sense the amount of the lateral displacement.

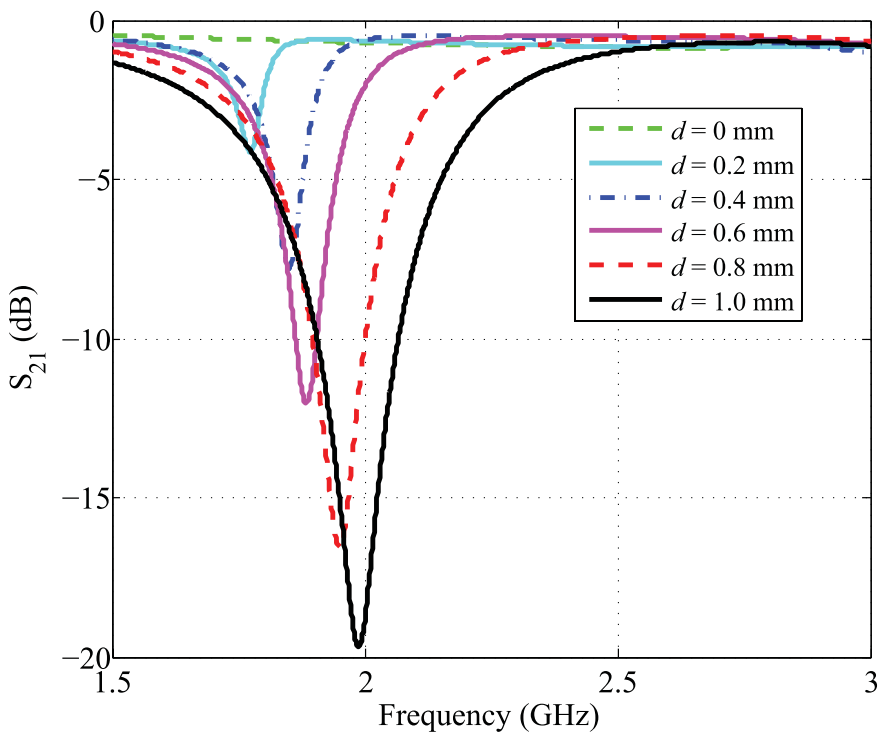

Fig. 3. Simulated transmission coefficient of the displacement sensor based on the diamond-shaped SRR (Fig. 2) for different values of displacement of the SRR from $d=0-1.0 \mathrm{~mm}$ in steps of $0.2 \mathrm{~mm}$.

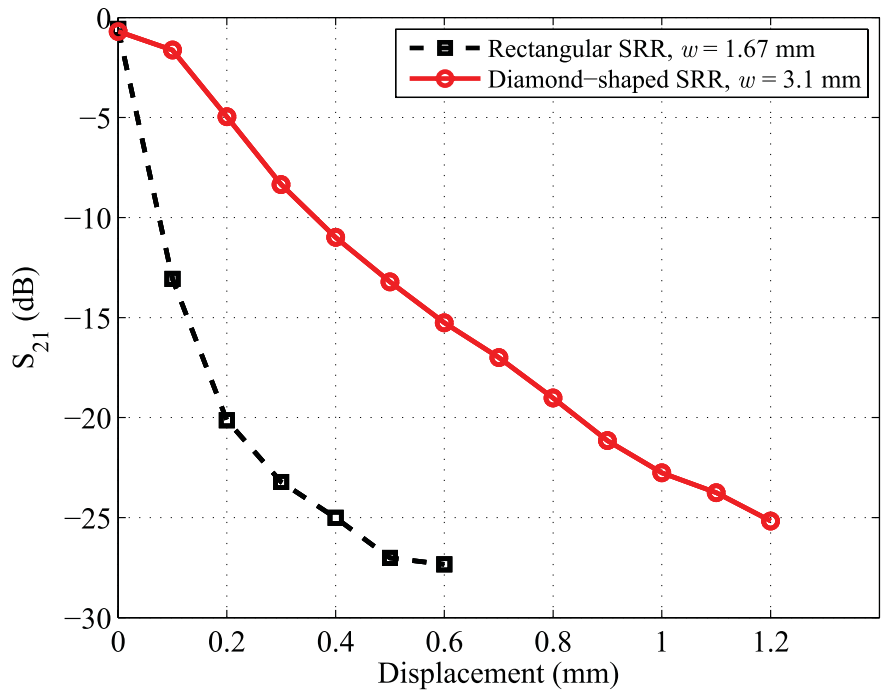

Fig. 4. Comparison between the linearity and dynamic range of displacement sensor based on rectangular-shaped double-ring SRR and those of the displacement sensor based on the diamond-shaped SRR.

Fig. 4 compares the notch magnitude versus the lateral displacement of the SRR in two different sensors, namely the displacement sensor based on the rectangular SRR (black dashed line) and the displacement sensor based on the diamondshaped SRR (red solid line). It is clear from the simulation results that even though the sensor with the rectangular-shaped SRR has a high sensitivity for small values of the lateral displacement it suffers from limited dynamic range of about $0.6 \mathrm{~mm}$. Furthermore, as mentioned earlier in this section, its linear behaviour is limited to a small range of displacement, approximately in the order of the CPW slot's width, $s=$ $0.2 \mathrm{~mm}$. In contrast, the sensor based on the diamond-shaped SRR benefits from a higher dynamic range of $1.2 \mathrm{~mm}$ and superior linearity within its dynamic range.

In short, the simulation results show that using a diamondshaped SRR instead of rectangular-shaped SRR adds one 


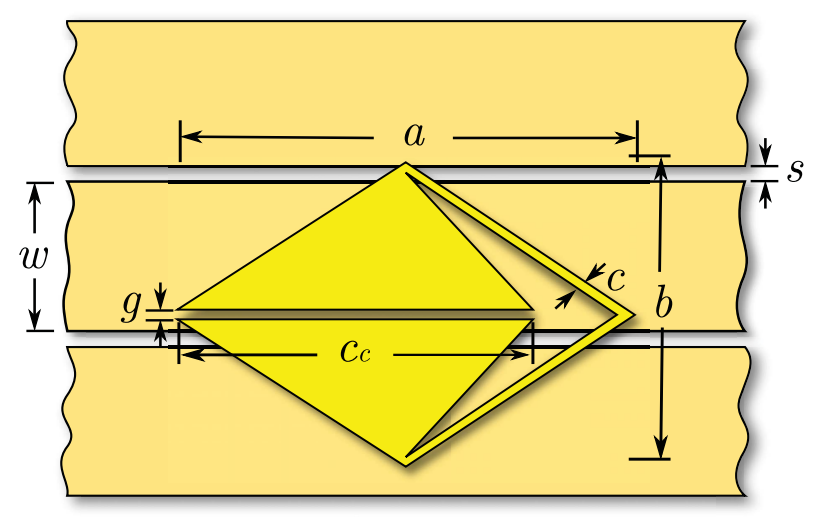

Fig. 5. Bottom view of coplanar waveguide loaded with a tapered diamondshaped split ring resonator.

degree of freedom to trade off sensitivity for dynamic range and linearity. In other words, the vertical diagonal of the diamond-shaped SRR $b$ along with the width of the CPW's signal strip can be adjusted in order to achieve a linear displacement sensor with desired dynamic range or sensitivity.

\section{B. Shift in Resonant Frequency}

The second limitation of the displacement sensor based on the rectangular SRR is that the displacement of the SRR not only changes the notch depth but also the resonant frequency. As can be seen in Fig. 3, this is also the limitation of the diamond-shaped sensor proposed in the previous subsection. The displacement sensor design is further improved in the following so that the resonant frequency remains fixed allowing the amount of displacement to be measured based on the notch magnitude in one fixed resonant frequency. This is an important improvement since the sensor can then be operated at a single frequency and bypass the need for a sophisticated frequency sweeping microwave source and measurement system.

It is worth recalling that in the quasistatic limit the SRR's fundamental resonant frequency can be approximated from the SRR's circuit model, which is a parallel LC resonator [27]. In such circumstances, the equivalent inductance of the SRR can be calculated from the inductance of a single ring with the average dimensions of the SRR and the equivalent capacitance can be calculated from the capacitance of the split [28]. However, in the case of the displacement sensor, the magnetic flux passing through the SRR surface is not uniform. As shown in Fig. 3, this results in a shift of the resonant frequency, which depends on the amount of displacement.

Since the resonant frequency is dependent on the equivalent inductance and capacitance of the SRR, it can be shown that a decrease in the horizontal diagonal $b$ of the SRR can be used to reduce the equivalent inductance of the SRR. This will minimize the effect of the non-uniformity of the magnetic flux passing through the SRR surface, and consequently diminish the shift in the resonant frequency. However, as stated above, a decrease in the diagonals of the SRR degrades the dynamic range and linearity of the sensor. Furthermore, decreasing the equivalent inductance of the SRR by decreasing $b$ pushes the

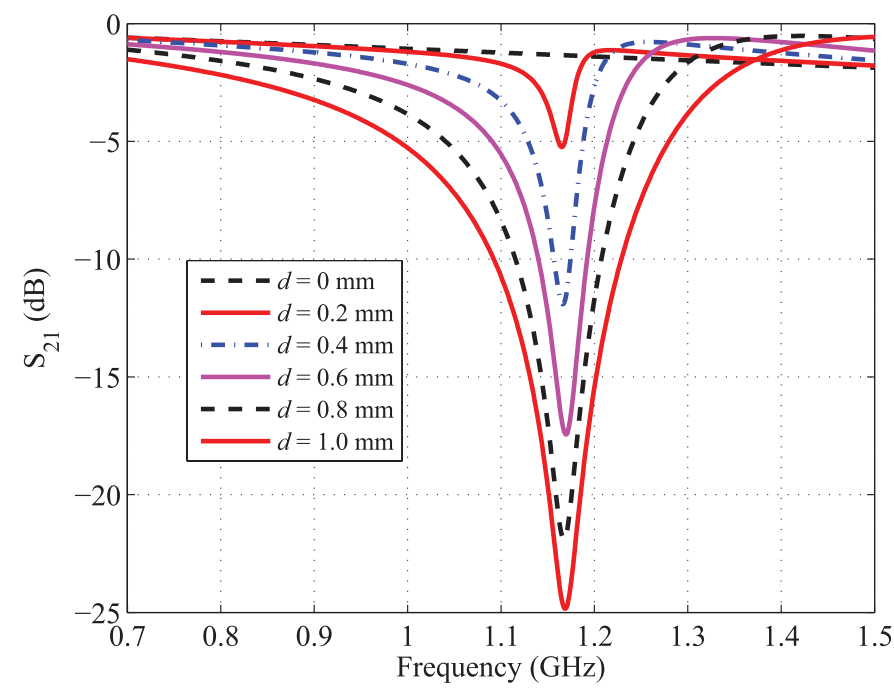

Fig. 6. Simulated transmission coefficient of the displacement sensor based on the tapered diamond-shaped SRR (Fig. 5) for different values of displacement of the SRR.

operating frequency of the sensor to high frequencies, which is generally not desirable in sensory applications.

Alternatively, tapering and widening the SRR's metallic strip, as illustrated in Fig. 5, can be adjusted to precisely compensate for the spatial non-uniformity of the magnetic flux passing through the SRR surface and consequently the shift in the resonant frequency is minimized. Furthermore, as will be shown later in this section, this also reduces the operating frequency of the displacement sensor by increasing the SRR's equivalent capacitance.

Fig. 5 depicts the bottom view of the proposed tapered diamond-shaped SRR. For 3D electromagnetic simulation the substrate properties $\left(\epsilon_{r}=10.2\right.$, thickness of $\left.0.127 \mathrm{~mm}\right)$, the CPW dimensions ( $w=3.1 \mathrm{~mm}, s=0.3 \mathrm{~mm})$ and the outer diamond shape ( $a=10 \mathrm{~mm}, b=6 \mathrm{~mm}$, and $c=g=0.2 \mathrm{~mm}$ ) remain unchanged. The only difference is in the width of the SRR's metallic strip on both sides of the SRR's split, which is optimized to be $c_{c}=8 \mathrm{~mm}$ to achieve a fixed resonant frequency.

Fig. 6 depicts the simulated transmission coefficients versus frequency for the displacement sensor based on the proposed tapered diamond-shaped SRR for different values of the lateral displacement of the SRR from $d=0 \mathrm{~mm}$ to $d=1 \mathrm{~mm}$ with steps of $0.2 \mathrm{~mm}$. The figure, clearly illustrates that the shift in the resonant frequency can be minimized through optimization of the parameter $c_{c}$. In that cases, all resonances occur at $1.17 \mathrm{GHz}$ independently of the displacement.

As mentioned earlier in this section, an increase in $c_{c}$ also increases the SRR's equivalent capacitance. Thus, from the comparison between the resonant frequency in Fig. 6, which is $1.17 \mathrm{GHz}$, and those in Fig. 3, which are higher than $1.75 \mathrm{GHz}$, it can be seen that the proposed tapered shape of the SRR pushes the resonant frequencies to lower frequencies without increasing the overall size of the SRR. That means that, in addition to having a fixed resonant frequency, the displacement sensor based on the tapered diamond-shaped SRR also benefits from a lower operating frequency. 


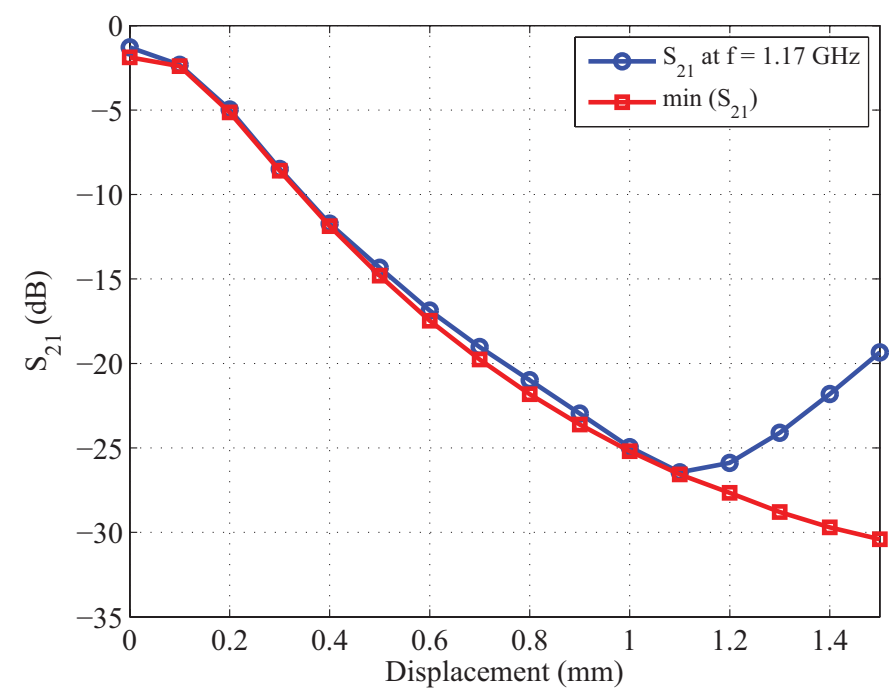

Fig. 7. Simulated response of the displacement sensor based on the tapered diamond-shaped SRR corresponding to notch magnitude. Red dashed line: at its minimum. Blue solid line: at the fixed frequency $f=1.17 \mathrm{GHz}$.

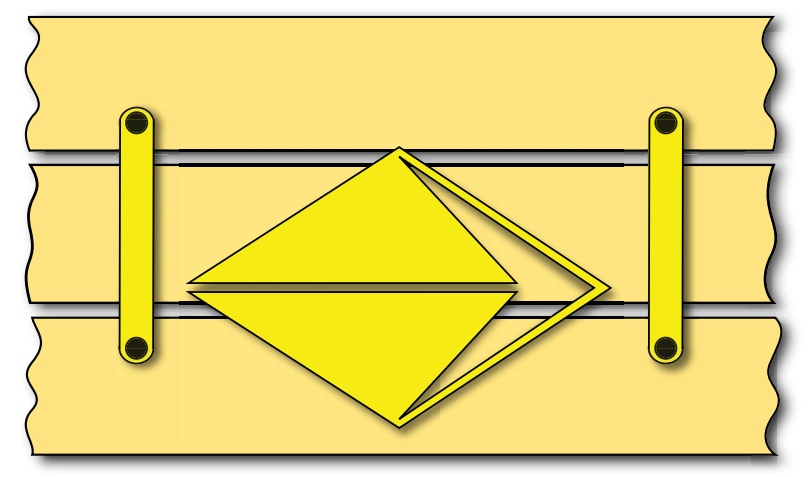

Fig. 8. Slotline mode can be suppressed by adding air-bridges along the CPW structure on both sides of the SRR to connect two ground planes and consequently equalize the voltages on two ground planes.

Fig. 7 depicts the simulated response of the tapered diamond-shaped sensor presented in Fig. 5 versus the lateral displacement of the SRR. The red line represents the notch magnitude in the transmission coefficient of the structure versus the lateral displacement of the SRR, and the blue line depicts the transmission coefficient of the structure at the fixed frequency $f=1.17 \mathrm{GHz}$. As clearly seen in the figure, for the SRR displacement less than $1.1 \mathrm{~mm}$ the blue line is aligned with the red line, indicating a dynamic range of $1.1 \mathrm{~mm}$, when it is used at the fixed frequency of $1.17 \mathrm{GHz}$. Furthermore, the sensor can achieve wider dynamic range of $1.5 \mathrm{~mm}$ if it is not used at a fixed frequency, i.e along with a frequency sweeping measurement system such as a network analyzer.

\section{Slotline Mode}

It is well known that any asymmetry and/or discontinuity in a CPW can excite the slotline mode (odd mode) in the line. For instance, the slotline mode is excited in a CPW at right-angle bends or T-junctions [29], [30]. It is worth mentioning that in all types of displacement sensors described in the previous

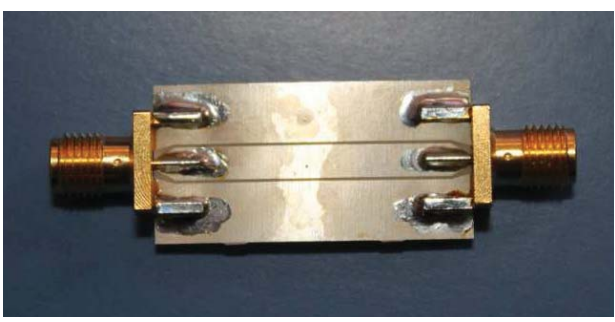

(a)

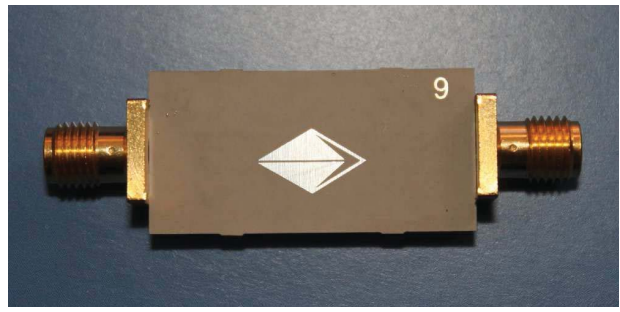

(b)

Fig. 9. Photograph of a sample of the fabricated sensors. (a) Top view of the CPW. (b) SRR on the back side substrate, which is displaced by $0.9 \mathrm{~mm}$ from the symmetry plane of the CPW.

sections, once the SRR is displaced from the symmetry plane of the CPW, the slotline mode is excited due to the asymmetry caused by the displaced SRR. As a result, the magnitude of the transmission notch becomes dependent on the length of the CPW. In all the presented simulations, the CPW's length is set to $30 \mathrm{~mm}$ and the CPW is directly connected to SMA coaxial connectors. In these cases, the length of the line becomes relevant as the slotline mode is suppressed by the coaxial connectors at both ends of the CPW. Nevertheless, in case where a longer length of CPW would be required, the propagation of the slotline mode can be suppressed by adding air-bridges along the $\mathrm{CPW}$ structure on both sides of the SRR, as shown in Fig. 8, to connect two ground planes and consequently equalize the voltages on two ground planes of the CPW [31].

\section{EXPERIMENTAL RESULTS}

Experimental results are presented to validate the SRR shape variation concept and electromagnetic simulation results of the previous sections. Due to the high sensitivity of the proposed sensor to small values of the SRR lateral displacement it is technically complex to perform an experimental validation by fabricating the structure with moving SRR. Thus, instead of a moveable SRR, several devices with different values of the SRR displacement are fabricated and measured as a proof-ofconcept.

Fig. 9 depicts the photograph of the top and bottom view of one of the fabricated samples with $d=0.9 \mathrm{~mm}$ displacement from the symmetry plane of CPW. The material used and the dimensions of the prototype corresponds to the simulation characteristic given in Section III.B.

Fig. 10 depicts the measured transmission coefficients of the sensor based on the tapered SRR for several values of the SRR's displacement. The figure shows a fixed resonant frequency at $f=1.13 \mathrm{GHz}$, which is in reasonable agreement with the simulation results shown in Fig. 6. The small 


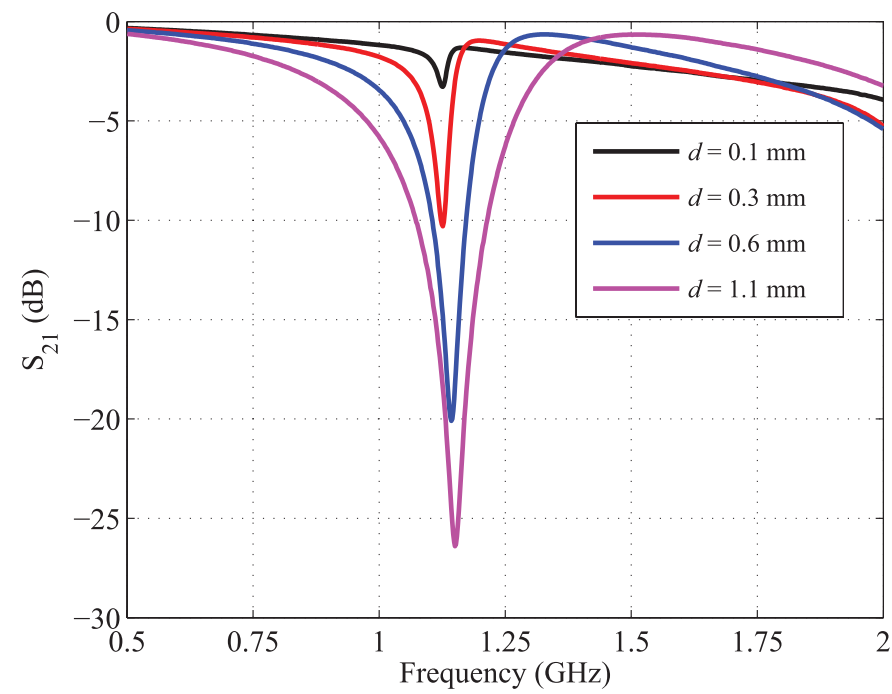

Fig. 10. Measured transmission coefficients of the sensor based on the tapered diamond-shaped SRR for different values of the SRR's displacement. The measurements are not as well aligned as the simulation in Fig. 6 because of the device-to-device variations. This is a consequence of each displacement requiring a separate device for this proof-of-concept. A movable SRR fabricated in MEMS technology will not exhibit this problem.

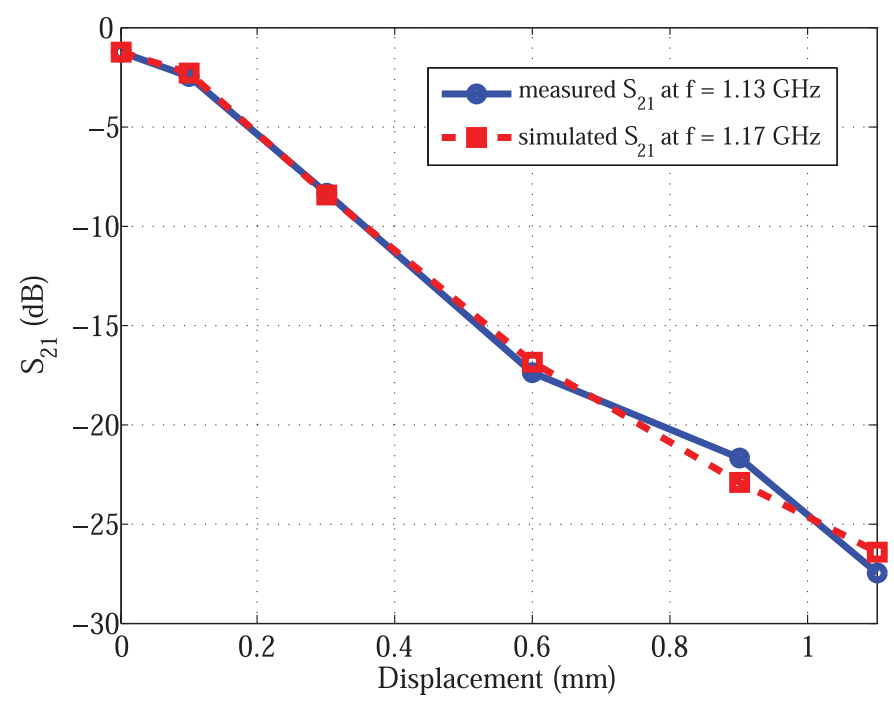

Fig. 11. Comparison between the transmission coefficient of the tapered diamond-shaped sensor versus the lateral displacement. Blue solid line: of the SRR measured at fixed frequency $f=1.13 \mathrm{GHz}$. Red dashed line: of the simulation results at fixed frequency $f=1.17 \mathrm{GHz}$.

difference between the measured and simulated resonant frequency can be attributed to variations in the substrate relative permittivity.

These results demonstrate that unlike in the case of the sensor based on the uniform diamond-shaped SRR (and rectangular SRR), the resonance in the sensor based on the tapered SRR occurs as predicted at a fixed frequency $f=1.13 \mathrm{GHz}$. This also validates the prediction that in addition to having a fixed resonant frequency, while maintaining a compact size, the displacement sensor based on the tapered diamond-shaped SRR also benefits from a lower operating frequency.

In Fig. 11, the measured transmission coefficient of the tapered diamond-shaped sensor versus the lateral displacement

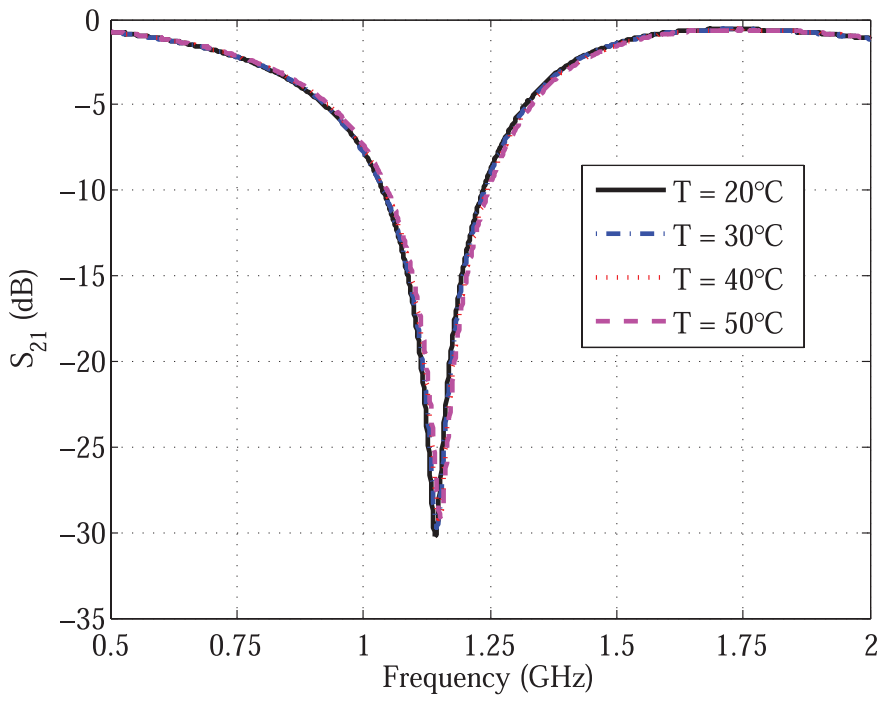

Fig. 12. Measured transmission coefficient of a 1.1-mm displaced tapered diamond-shaped sensor for different temperatures form $20{ }^{\circ} \mathrm{C}$ to $50{ }^{\circ} \mathrm{C}$ with steps of $10{ }^{\circ} \mathrm{C}$.

of the SRR at fixed frequency $f=1.13 \mathrm{GHz}$ (blue solid line) is compared with that of the simulation results at fixed frequency $f=1.17 \mathrm{GHz}$ (red dashed line). The graph shows good agreement between measured and simulated results.

To investigate the effect of the thermal expansions of the substrate and metallic layers the transmission coefficient of a particular sample, specifically with $1.1 \mathrm{~mm}$ displacement, is measured at different temperatures from $20^{\circ} \mathrm{C}$ to $50{ }^{\circ} \mathrm{C}$. The measured results, depicted in Fig. 12, show that for a $30{ }^{\circ} \mathrm{C}$ increase in the temperature, the measurement yields only a $1 \%$ increase in the resonant frequency of the structure and less than a $1 \mathrm{~dB}$ decrease in the depth of resonance. This demonstrates that the sensor has a good thermal stability.

\section{CONCLUSION}

This work has presented a displacement sensor based on a diamond-shaped tapered SRR. The proposed shape modifications of the SRR have enabled us to improve the dynamic range of the sensor from $0.6 \mathrm{~mm}$ to $1.1 \mathrm{~mm}$, which corresponds to an improvement of more than $80 \%$. Also, compared to the displacement sensor based on a rectangular-shaped $\mathrm{SRR}$, the proposed sensor has superior linearity. It is also shown that the undesirable shift in the resonant frequency in the characteristic of previous designs can be suppressed with an optimized tapered diamond-shaped SRR. This is a significant improvement since the sensor does not require a frequency sweeping microwave source, but can be operated as inexpensive single frequency system. Compared to the previously published displacement sensors, the proposed sensor also benefits from a lower operating frequency, which is generally desirable in sensing applications. Good agreement between simulation and measurement results validates the design principles. 


\section{ACKNOWLEDGMENT}

The authors would like to acknowledge P. Simcik for his technical assistance.

\section{REFERENCES}

[1] J. J. Yang, M. Huang, and J. Sun, "Double negative metamaterial sensor based on microring resonator," IEEE Sensors J., vol. 11, no. 10, pp. 2254-2259, Oct. 2011.

[2] S. A. Taya and M. M. Shabat, "Sensitivity enhancement in optical waveguide sensors using metamaterials," Appl. Phys. A, Mater. Sci. Process., vol. 103, no. 3, pp. 611-614, 2011.

[3] J. Pendry, D. Schurig, and D. Smith, "Controlling electromagnetic fields," Science, vol. 312, no. 5781, pp. 1780-1782, 2006.

[4] P. Alitalo and S. Tretyakov, "Electromagnetic cloaking with metamaterials," Mater. Today, vol. 12, no. 3, pp. 22-29, 2009.

[5] S. Enoch, G. Tayeb, P. Sabouroux, N. Guérin, and P. Vincent, "A metamaterial for directive emission," Phys. Rev. Lett., vol. 89, no. 21, pp. 213902-1-213902-4, 2002.

[6] A. Dubinov and L. Mytareva, "Invisible cloaking of material bodies using the wave flow method," Phys.-Uspekhi, vol. 53, no. 5, pp. 455-479, 2010.

[7] A. K. Horestani, Z. Shaterian, S. Al-Sarawi, and D. Abbott, "High quality factor mm-wave coplanar strip resonator based on split ring resonators," in Proc. 36th Int. Conf. Infr., Millim. Terahertz Waves, 2011, pp. $1-2$.

[8] A. K. Horestani, Z. Shaterian, W. Withayachumnankul, C. Fumeaux, S. Al-Sarawi, and D. Abbott, "Compact wideband filter element based on complementary split-ring resonators," Proc. SPIE, Smart Nano-Micro Mater. Devices, vol. 8204, pp. 820431-1-820431-7, Sep. 2011.

[9] J. Pendry, "Negative refraction makes a perfect lens," Phys. Rev. Lett., vol. 85, no. 18, pp. 3966-3969, 2000.

[10] M. Huang and J. Yang, "Microwave sensor using metamaterials," in Wave Propagation. Rijeka, Croatia: InTech, 2011, pp. 13-36.

[11] M. Huang, J. Yang, J. Sun, J. Shi, and J. Peng, "Modelling and analysis of $\Omega$-shaped double negative material-assisted microwave sensor," $J$. Infr., Millim. Terahertz Waves, vol. 30, no. 11, pp. 1131-1138, 2009.

[12] J. Yang, M. Huang, Z. Xiao, and J. Peng, "Simulation and analysis of asymmetric metamaterial resonator-assisted microwave sensor," Mod. Phys. Lett. B, vol. 24, no. 12, pp. 1207-1215, 2010.

[13] W. Withayachumnankul, K. Jaruwongrungsee, C. Fumeaux, and D. Abbott, "Metamaterial-inspired multichannel thin-film sensor," IEEE Sensors J., vol. 12, no. 5, pp. 1455-1458, May 2012.

[14] N. I. Zheludev, "The road ahead for metamaterials," Science, vol. 328, no. 5978 , pp. $582-583,2010$.

[15] Z. Jakic, O. Jakic, Z. Djuric, and C. Kment, "A consideration of the use of metamaterials for sensing applications: Field fluctuations and ultimate performance," J. Opt. A, Pure Appl. Opt., vol. 9, no. 9, pp. S377-S384, 2007.

[16] S. He, Y. Jin, Z. Ruan, and J. Kuang, "On subwavelength and open resonators involving metamaterials of negative refraction index," New J. Phys., vol. 7, no. 1, p. 210, Oct. 2005.

[17] E. Cubukcu, S. Zhang, Y.-S. Park, G. Bartal, and X. Zhang, "Split ring resonator sensors for infrared detection of single molecular monolayers," Appl. Phys. Lett., vol. 95, no. 4, pp. 043113-1-043113-3, 2009.

[18] P. Pattnaik, B. Vijayaaditya, T. Srinivas, and A. Selvarajan, "Optical MEMS pressure sensor using ring resonator on a circular diaphragm," in Proc. Int. Conf. MEMS, NANO Smart Syst., 2005, pp. 277-280.

[19] R. Melik, E. Unal, N. K. Perkgoz, C. Puttlitz, and H. V. Demir, "Metamaterial-based wireless strain sensors," Appl. Phys. Lett., vol. 95, no. 1, pp. 011106-1-011106-3, 2009.

[20] A. Alù and N. Engheta, "Dielectric sensing in $\epsilon$-near-zero narrow waveguide channels," Phys. Rev. B, vol. 78, no. 4, pp. 045102-1-045102-5, 2008

[21] D. Shreiber, M. Gupta, and R. Cravey, "Comparative study of 1-D and 2-D metamaterial lens for microwave nondestructive evaluation of dielectric materials," Sens. Actuators A, Phys., vol. 165, no. 2, pp. 256-260, 2011.

[22] J. Naqui, M. Durán-Sindreu, and F. Martín, "Novel sensors based on the symmetry properties of split ring resonators (SRRs)," Sensors, vol. 11, no. 8, pp. 7545-7553, 2011.

[23] F. Martin, F. Falcone, J. Bonache, R. Marques, and M. Sorolla, "Miniaturized coplanar waveguide stop band filters based on multiple tuned split ring resonators," IEEE Microw. Wireless Compon. Lett., vol. 13, no. 12 , pp. 511-513, Dec. 2003.
[24] J. Garcia-Garcia, F. Martin, F. Falcone, J. Bonache, I. Gil, T. Lopetegi, M. Laso, M. Sorolla, and R. Marques, "Spurious passband suppression in microstrip coupled line band pass filters by means of split ring resonators," IEEE Microw. Wireless Compon. Lett., vol. 14, no. 9, pp. 416-418, Sep. 2004.

[25] F. Martín, J. Bonache, F. Falcone, M. Sorolla, and R. Marqués, "Split ring resonator-based left-handed coplanar waveguide," Appl. Phys. Lett., vol. 83, no. 22, pp. 4652-4654, 2003.

[26] X. He, Y. Wang, J. Wang, and T. Gui, "Thin-film sensor based tip-shaped split ring resonator metamaterial for microwave application," Microsyst. Technol., vol. 16, no. 10, pp. 1735-1739, 2010.

[27] R. Marqués, F. Medina, and R. Rafii-El-Idrissi, "Role of bianisotropy in negative permeability and left-handed metamaterials," Phys. Rev. B, vol. 65 , no. 14 , pp. $144440-1-144440-6,2002$.

[28] M. Shamonin, E. Shamonina, V. Kalinin, and L. Solymar, "Resonant frequencies of a split-ring resonator: Analytical solutions and numerical simulations," Microw. Opt. Technol. Lett., vol. 44, no. 2, pp. 133-136, 2005.

[29] N. Dib, M. Gupta, G. Ponchak, and L. Katehi, "Characterization of asymmetric coplanar waveguide discontinuities," IEEE Trans. Microw. Theory Tech., vol. 41, no. 9, pp. 1549-1558, Sep. 1993.

[30] R. Simons and G. Ponchak, "Modeling of some coplanar waveguide discontinuities," IEEE Trans. Microw. Theory Tech., vol. 36, no. 12, pp. 1796-1803, Dec. 1988

[31] G. Ponchak, J. Papapolymerou, and M. Tentzeris, "Excitation of coupled slotline mode in finite-ground CPW with unequal ground-plane widths," IEEE Trans. Microw. Theory Tech., vol. 53, no. 2, pp. 713-717, Feb. 2005.

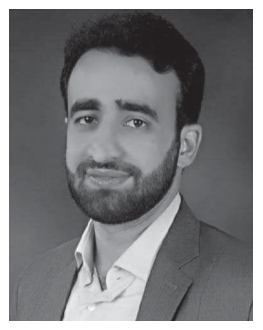

Ali Karami Horestani (S'09) was born in Isfahan, Iran. He received the B.Eng. and M.Eng. degrees in electrical and electronics engineering from the University of Shiraz, Shiraz, Iran, and the University of Shahid Beheshti, Tehran, Iran, in 2003 and 2006, respectively. He is currently pursuing the $\mathrm{Ph} . \mathrm{D}$. degree in engineering with the University of Adelaide, Adelaide, Australia, in microwave and millimetre wave circuit design.

His current research interests include MMIC design, microwave and millimeter devices and circuits, and metamaterials and their applications in planar circuits.

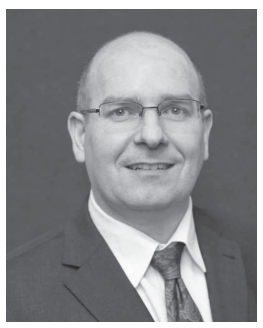

Christophe Fumeaux (M'03-SM'09) received the Diploma and Ph.D. degrees in physics from ETH Zurich, Zurich, Switzerland, in 1992 and 1997, respectively.

He was a Post-Doctoral Researcher with the School of Optics, University of Central Florida, Orlando, from 1998 to 2000. In 2000, he joined the Swiss Federal Office of Metrology, Bern, Switzerland, as a Scientific Staff Member. From 2001 to 2008, he was a Research Associate and Group Leader with the Laboratory for Electromagnetic Fields and Microwave Electronics, ETH, Zurich. In 2008, he joined the School of Electrical and Electronic Engineering, University of Adelaide, Adelaide, Australia, as an Associate Professor. Since 2011, he has been a Future Fellow of the Australian Research Council. His current research interests include computational electromagnetics, antenna engineering, $\mathrm{THz}$ technology, and the application of RF design principles to optical micro/nano-structures.

Dr. Fumeaux is an Associate Editor for the IEEE TRANSACTIONS ON Microwave Theory AND Techniques. He was the Chairman of the IEEE Swiss Joint Chapter on Microwave Theory and Techniques (MTT-S), Antennas and Propagation (AP-S), and Electromagnetic Compatibility (EMC) from 2006 to 2008. Since 2010, he has been the Chairman of the IEEE South Australia AP-S/MTT-S Chapter. He was the recipient of the ETH Silver Medal of Excellence for his doctoral dissertation. He was the corecipient of the 2004 Outstanding Paper Award of the Applied Computational Electromagnetics Society and the Best Symposium Paper Award at the 2012 Asia-Pacific International Symposium on Electromagnetic Compatibility. 


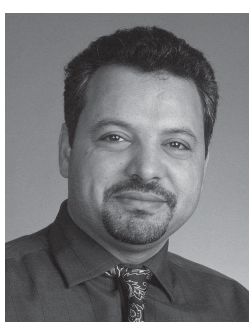

Said F. Al-Sarawi (M'92) received the general certificate in marine radio communication and the B.Eng. degree (Hons.) in marine electronics and communication in 1987 and 1990, respectively, and the $\mathrm{Ph} . \mathrm{D}$. degree in mixed analog and digital circuit design techniques for smart wireless systems with special commendation from the Department of Electrical and Electronic Engineering, in 2003, and the Graduate Certificate degree in education (higher education) in 2006, all from the Arab Academy for Science and Technology, Alexandria, Egypt.

$\mathrm{He}$ is currently the director of the Centre for Biomedical Engineering and a Founding Member of Education Research Group of Adelaide, University of Adelaide, Adelaide, Australia. His current research interests include design techniques for mixed signal systems in CMOS and optoelectronic technologies for high performance radio transceivers, low power and low voltage radio frequency identification systems, data converters, mixed signal design, and micro-electro-mechanical systems for biomedical applications.

Dr. Al-Sarawi received the University of Adelaide Alumni Postgraduate Medal (formerly Culross) for outstanding academic merit at the postgraduate level and the Commonwealth Postgraduate Research Award (Industry).

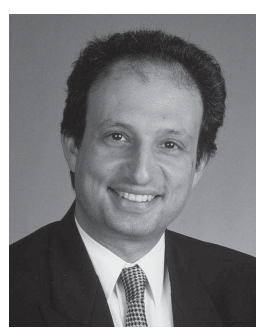

Derek Abbott (M'85-SM'99-F'05) was born in South Kensington, London, U.K., on May 3, 1960. $\mathrm{He}$ received the B.Sc. (Hons.) degree in physics from the Loughborough University of Technology, Leicestershire, U.K., in 1982, and the Ph.D. degree (with commendation) in electrical and electronic engineering from The University of Adelaide, Adelaide, Australia, in 1995, under K. Eshraghian and B. R. Davis.

He has led a number of research programs in the imaging arena, ranging from the optical to infrared to millimeter wave to T-ray (terahertz gap) regimes. From 1978 to 1986, he was with the GEC Hirst Research Centre, London, in the area of visible and infrared image sensors. His expertise spans VLSI design, optoelectronics, device physics, and noise; where he has worked with nMOS, CMOS, SOS, $\mathrm{CCD}, \mathrm{GaAs}$, and vacuum microelectronic technologies. He was with Austek Microsystems, Technology Park, South Australia, in 1986. Since 1987, he has been with The University of Adelaide, where he is currently a full Professor with the School of Electrical and Electronic Engineering. He has appeared on national and international television and radio and has also received scientific reportage in New Scientist, The Sciences, Scientific American, Nature, The New York Times, and Sciences et Avenir. He holds over 300 publications/patents and has been an invited speaker at over 80 institutions, including Princeton, NJ; MIT, MA; Santa Fe Institute, NM; Los Alamos National Laboratories, NM; Cambridge, UK; and EPFL, Lausanne, Switzerland. He has co-authored the book Stochastic Resonance (Cambridge University Press) and co-edited the book Quantum Aspects of Life (Imperial College Press).

Prof. Abbott is a fellow of the Institute of Physics, with honorary life membership. He received the GEC Bursary in 1977, the Stephen Cole the Elder Prize in 1998, the E. R. H. Tiekink Memorial Award in 2002, the SPIE Scholarship Award for Optical Engineering and Science in 2003, the South Australian Tall Poppy Award for Science in 2004, and the Premier's SA Great Award in Science and Technology for outstanding contributions to South Australia in 2004. He has served as an Editor and/or Guest Editor for a number of journals, including the IEEE Journal of Solid-State Circuits, Chaos (AIP), Smart Structures and Materials (IOP), Journal of Optics B $(I O P)$, Microelectronics Journal (Elsevier), Fluctuation Noise Letters (World Scientific), and is currently on the Editorial Boards of Proceeding of the IEEE and the IEEE Photonics. He has served on a number of IEEE technical program committees, including the IEEE APCCS and the IEEE GaAs IC Symposium. 